\title{
Methods of the analysis of motion of small space vehicles around the centre of masses at deployment of space tether system
}

\author{
Yuriy Zabolotnov* and Oleg Naumov \\ Department of Software Systems, \\ Faculty of Computer Science, \\ The Samara Sate Aerospace University (National Research University), \\ 34 Moscow Highway, Samara, \\ The Samara Region, The Russian Federation \\ Email: yumza@yandex.ru \\ Email: olegnaumov6386@yandex.ru \\ *Corresponding author
}

\begin{abstract}
This paper studies analytical and numerical methods of the analysis of spatial motion concerning centre of mass of small space vehicles at deployment of space tether system. Deployment of space tether system is executed from the base space vehicle. The set angular orientation of the base space vehicle concerning a local vertical is provided by means of own system of stabilisation. The small space vehicle (or freight) represents a descent capsule returned to the Earth or the space vehicle transferred into higher orbit. It is supposed that the weight of the base space vehicle is much more than weight of freight. The angular position of freight is described by classical Euler's angles determined concerning a direction of a tether. Analytical methods of the analysis of angular motion are based on an averaging method (for small angles of nutation) and on a method integral of manifolds which allows to investigate the nonlinear equations of angular motion of freight. The major factors influencing stability of angular motion of freight concerning a direction of a tether are considered. Results of numerical modelling of angular motion of the small space vehicle are presented.
\end{abstract}

Keywords: space vehicle; space tether system; STS; deployment; averaging method; stability; disturbances.

Reference to this paper should be made as follows: Zabolotnov, Y. and Naumov, O. (2014) 'Methods of the analysis of motion of small space vehicles around the centre of masses at deployment of space tether system', Int. J. Space Science and Engineering, Vol. 2, No. 4, pp.305-326.

Biographical notes: Yuriy Zabolotnov is a Professor at Samara State Aerospace University (Russian Federation). He received his scientific degree of DrSciTech in 1996 and was appointed as professor in 1998. He has published more than 30 scientific articles and two monographs. His scientific interests include mechanics of space flight, dynamics of descent of space vehicles in atmosphere, dynamics and control motion of space tether systems, and asymptotic methods of mechanics. From 2004-2011, he was the Head of Department of Mathematics and Mechanics of the Samara State Aerospace University. He is a full member of Academy of Navigation and Control (St. Petersburg, Russia). 
Oleg Naumov is an Associate Professor at Samara State Aerospace University. $\mathrm{He}$ received his $\mathrm{PhD}$ degree in 2012. His research interests include the dynamics of space tether systems dynamics of rigid body and computational mathematics. Currently, he is working at the Department of Software Systems, Samara State Aerospace University.

This paper is a revised and expanded version of a paper entitled 'Analysis of the rotational motion of a body on an elastic tether' presented at the International Conference Scientific and Technological Experiments on Automated Space Vehicles and Small Satellites (SPEXP), Samara, Russia, 2-5 September 2008.

\section{Introduction}

Many authors dedicate great attention to the deployment of space tether systems (STSs). The given problem includes:

1 construction of nominal or ideal programmes of the deployment of STS in the set position

2 synthesis of algorithms of the stabilisation providing demanded quality of transients at deployment of STS

3 safety control at jettisoning of a payload from the base space vehicle (an exception of their collision) on an initial site of deployment of STS

4 damp of transversal and longitudinal oscillations in the course of deployment of STS

5 exception of resonant modes of the motion leading to instability of process of deployment of STS.

This list of tasks is not complete, since each experiment had its special task. Separate consideration is demanded also by electrodynamic tether systems, naturally, having features of deployment and operation owing to interaction with a magnetic field of the Earth (Zhong and Zhu, 2013).

Deploying the STS is a complex transient and dynamic process, the success of all space flight depends on the quality of design. It is important to know what movement is small spacecraft around the center of mass at deployment of the STS. In some tether experiments control of an angular position of tip bodies was executed, for example, it concerns missions OEDIPUS-A and C (Vigneron et al., 2000), conducted in 1989 and 1995. And the post-flight analysis has shown (Tyc and Han, 2001) that at implementation of mission OEDIPUSA the uncontrollable increase in angle of a deviation of a tip body from a tether direction was observed. In the given article the main disturbances and the factors influencing motion concerning centre of mass of the small space vehicle at deployment of STS are analysed. Here the development of system for big enough length of a tether (about 30 kilometres) is considered.

Technology return to Earth a small capsule with a nearly circular orbit with the use of STS has been implemented in the experiment YES2. The process of deployment in YES2 
experiment consisted of two phases. The first stage is a stage of rather slow extension of a tether with tether reduction in a vertical position. The second stage - a stage of fast deployment of STS in a final condition with the position of a tether rejected from a vertical for the message to a descent capsule of an additional braking impulse. At this stage, the law consisting of sites of fast boost and braking of a tether is used. Deployment programmes are realised by means of the gear of control by extension of the tether working with use of a principle of feedback (Kruijff, 2011).

Non-observance of limitations on angular motion of the small space vehicle can result in the course of deployment of STS in tether sagging (controllability loss), to formation of loops, to tether torsion, to tether winding on a tip body etc. All this can significantly complicate the fulfilment of the objectives of the mission.

Below, we consider a fairly complete analysis of the factors influencing the parameters of the angular motion of a spacecraft on a tether. Influence of the following factors is analysed:

1 the nominal programme of deployment of STS

2 parameters of a control system, the gear of control and tether characteristics

3 static and dynamic asymmetry of the small space vehicle.

For the analysis of influence of the nominal programme of deployment and asymmetry on motion of the small space vehicle asymptotic methods are used: an averaging method (Bogolyubov and Mitropolsky, 1963; Nayfeh, 1973) and a method of integral manifolds (Mitropolsky and Lykova, 1973; Hirsh et al., 1977). The analytical solutions describing angular motion of a tip body at small and considerable angles of nutation at slow change of force of a tension of a tether are received. The nutation angle in this case is an angle between a direction of a tether and a space vehicle centreline. The method of integral manifolds to determine the possibility of nonlinear resonances that leads to large perturbations in the rotational motion of the capsule.

In this article, the review and generalisation of results of the researches conducted by authors in the given direction from 2007 for 2013 is presented.

\section{Statement of the problem}

The motion of a spacecraft on a tether has its counterpart classical mechanical problem of the motion of a heavy rigid body about a fixed point with the replacement of the tether on the direction of the vertical direction. And in this case, it is assumed that the moment of the tether tension force is much larger than the other side (gravity, drag, and so on.), which can be attributed to perturbations. Consequently, there is a case close to the classical problem of the motion of the Lagrange top under the action of small perturbations. Therefore, at a constant tether tension for symmetric rigid body holds the unperturbed case, which is characterised by well-known first integrals of motion. All these are the basis for the application of asymptotic methods of analysis of perturbed oscillatory systems. 
Figure 1 Space tether system

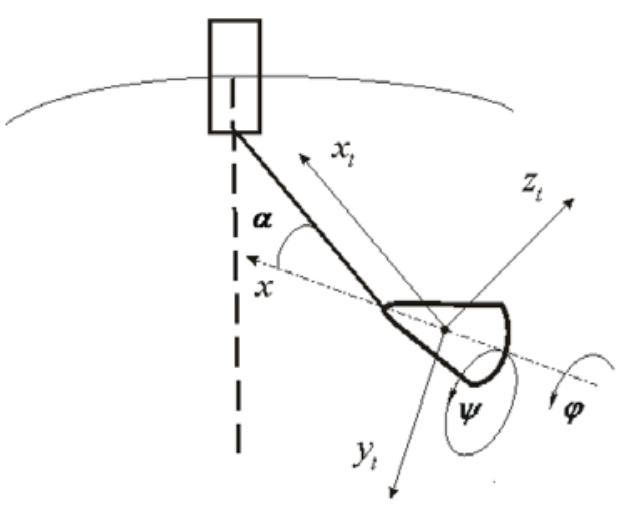

The position of a rigid body with respect to the direction of the tether is determined by the three Euler angles: precession $\psi$, nutation $\alpha$ and proper rotation $\varphi$ (Figure 1). It is assumed that the tether does not touch the surface of the cargo, which leads to a definite limitation on the size of the angle of nutation, depending on the geometry of the spacecraft. For example, the nutation angle cannot exceed $\pi / 2$ for a body close to a sphere (or spherical toe).

Euler angles are defined relative to the cargo's so-called tether system of coordinates $c x_{t} y_{t} z_{t}$, where $c$ - centre of mass of the cargo, axis $c x_{t}$ is parallel to the direction of the tether, axis $c y_{t}$ lies in the plane, 'the tether - local vertical' (local vertical drawn through the centre of mass of the base of the spacecraft), axis $c z_{t}$ completes the coordinate system to the right. Figure 2 shows the order and direction of the elementary rotations corresponding to the Euler angles $(\psi, \alpha, \varphi)$ and defines the transition between bound $c x y z$ and tethered coordinate systems. Figure 2 also shows the positions of the intermediate coordinate systems that are used below to write the equations of motion of cargo.

Figure 2 Euler angles

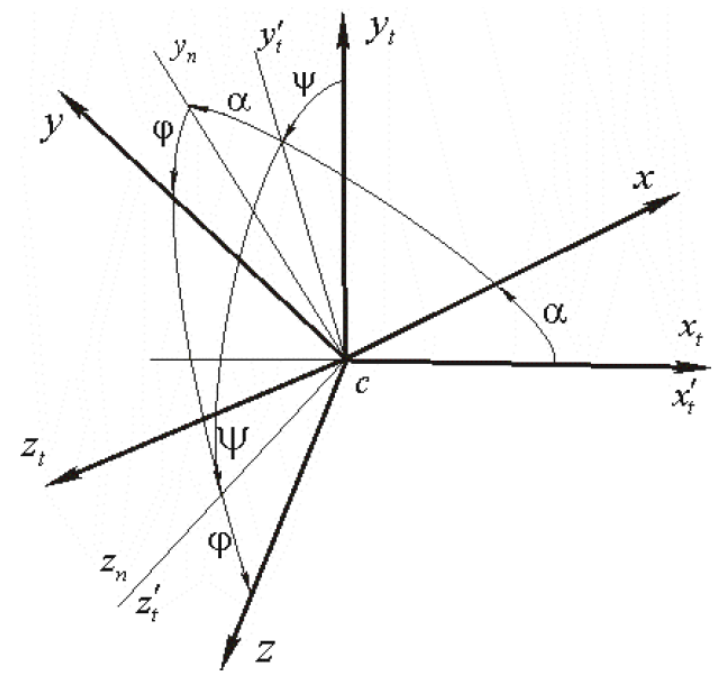


Movement of cargo to the direction of the tether described by classical dynamic and kinematic equations of Euler

$$
\begin{aligned}
& \frac{\tilde{d} \vec{K}_{c}}{d t}+\overrightarrow{\tilde{\omega}} \times \vec{K}_{c}=\vec{M}_{c} \\
& \frac{d \alpha}{d t}=\omega_{z} \cos \varphi+\omega_{y} \sin \varphi+\Delta \dot{\alpha} \\
& \frac{d \psi}{d t}=\left(\omega_{z} \sin \varphi-\omega_{y} \cos \varphi\right) / \sin \alpha+\Delta \dot{\psi} \\
& \frac{d \varphi}{d t}=\omega_{x}+\left(\omega_{y} \cos \varphi-\omega_{z} \sin \varphi\right) \operatorname{ctg} \alpha+\Delta \dot{\varphi}
\end{aligned}
$$

where $\vec{K}_{c}$ - moment of momentum vector of cargo, $\frac{\tilde{d} \vec{K}_{c}}{d t}-$ local derivative, $\overrightarrow{\tilde{\omega}}-$ angular velocity of rotation of the coordinate system on which the projected vector equation (1); $\vec{M}_{c}$ - principal moment of the external forces acting on the cargo; $\omega_{x}, \omega_{y}$, $\omega_{z}$ - components of the vector $\vec{\omega}$ rotational cargo in the coupled system of coordinates cxyz; $\Delta \dot{\alpha}, \Delta \dot{\varphi}, \Delta \dot{\psi}$ - amendments to derivatives of the Euler angles, arising due to the rotation of the coordinate system of tether $c x_{t} y_{t} z_{t}$.

Equation (1) is projected on different coordinate systems depending on the purposes of analysis.

Principal moment of the external forces $\vec{M}_{c}$ is the sum of

$$
\vec{M}_{c}=\vec{M}_{c t}+\Delta \vec{M}_{c}
$$

where $\vec{M}_{c t}=\Delta \vec{r} \times \vec{T}-$ moment on the force of the tether tension, $\Delta \vec{r}-$ vector defining the position of the point of attachment of a tether from the centre of mass of the cargo; $\vec{T}$ - tether tension force; $\Delta \vec{M}_{c}$ - vector sum of the small moments of forces (perturbations), acting on the cargo.

The strength of the tether is

$$
\vec{T}=\vec{T}_{n}+\Delta \vec{T}
$$

where $\vec{T}_{n}$ - nominal of the tether tension force, $\Delta \vec{T}$ - component of the tether tension force that depends on the operation of control, from the elongation of a tether, etc.

In a related system of coordinates of the vector $\Delta \vec{r}$ has the components $(\Delta x, \Delta y, \Delta z)$, where values $\Delta y, \Delta z$ determine the static cargo asymmetry. Dynamic asymmetry of a rigid body is characterised by the off-diagonal components of the inertia tensor, recorded in the associated coordinate system, as well as the difference between the axial moments of inertia $J_{z}-J_{y}$. For statically and dynamically symmetric body have $\Delta y=\Delta z=0, J_{y}=J_{z}$ $=J \neq J_{x}, J_{x y}=J_{x z}=J_{y z}=0$, where $J_{x y}, J_{x z}, J_{y z}$ - the centrifugal moments of inertia.

The equations describing the motion of the mass centres of the end-bodies are written in one form or another, depending on the objectives of the study (Zabolotnov, 2013), taking into account the geometrical relationships between different parts of STS. 
In accordance with the mathematical model (1 to 4$)$ analyses the impact of the slow change of the nominal tensile force $\vec{T}_{n}$ and other small perturbations dependent variables $\Delta \vec{T}, \Delta y, \Delta z, J_{z}-J_{y}, J_{x y}, J_{x z}, J_{y z}, \Delta \vec{M}_{c}$, to the rotational motion of a small spacecraft to deploy STS.

\section{Analysis of the rotational motion of the small spacecraft at small angles nutation}

Using the assumption of smallness of the nutation angle allows for the most comprehensive asymptotic analysis of stability of the angular motion of a small spacecraft with respect to the direction of the tether, although it has limited use.

To obtain the equations of motion that are convenient for the asymptotic analysis, the vector equation (1) is projected on the axis $c x, c y_{t}, c z_{t}$.

$$
\begin{aligned}
& \frac{d K_{x}}{d t}+K_{z} \omega_{y}-K_{y} \omega_{z}=M_{x} \\
& \frac{d K_{y t}}{d t}+K_{x t} \tilde{\omega}_{z t}-K_{z t} \tilde{\omega}_{x t}=M_{y t} \\
& \frac{d K_{z t}}{d t}+K_{y t} \tilde{\omega}_{x t}-K_{x t} \tilde{\omega}_{y t}=M_{z t}
\end{aligned}
$$

where $\tilde{\omega}_{x t}, \tilde{\omega}_{y t}, \tilde{\omega}_{z t}$ - components of the angular velocity of rotation of the tether system of coordinates (Zabolotnov and Naumov, 2012).

Equations (8)-(9) through the introduction of complex values $K_{y t}+i K_{z t}, M_{y t}+i M_{z t}$ are reduced to a single equation for the complex angle of nutation $\xi=i \alpha e^{i \cdot \psi}$.

$$
\frac{d^{2} \xi}{d t^{2}}-i \bar{J}_{x} \omega_{x} \frac{d \xi}{d t}+\omega_{0}^{2}(r) \xi=\varepsilon F\left(r, \xi, \omega_{x}, \Phi\right)
$$

where functions $F\left(r, \xi, \omega_{x}, \Phi\right)$ determine the effect of small perturbations in the system, $\bar{J}_{x}=J_{x} / J, \varepsilon-$ it is small parameter of the problem, $\Phi=\varphi+\psi, \omega_{0}^{2}(r)=\Delta x T_{n}(r) / J$, $r-$ it is vector slowly changing variables that affect the nominal deployment programme STS.

Equation (10) need to attach the equations for the variables $\omega_{x}$ and $\Phi$

$$
\begin{aligned}
\frac{d \omega_{x}}{d t} & =\varepsilon f\left(r, \xi, \omega_{x}, \Phi\right) \\
\frac{d \Phi}{d t} & =\omega_{x}
\end{aligned}
$$

This form of the equations (10)-(12) presented in the paper (Zabolotnov and Naumov, 2012).

A similar form of the equations of rotational motion (10)-(12) has been used previously in the works belonging to the adjacent areas of solid mechanics. Thus, the 
complex form of the equations of motion used in the task, dedicated input dynamics of the spacecraft into the atmosphere (Nayfeh, 1973; Zabolotnov, 1994).

It is using the method of averaging to obtain the asymptotic solutions of the system (10)-(12). System (10)-(12) is the unperturbed solution in the absence of asymmetry for a constant tensile force $T=$ const

$$
\begin{aligned}
& \xi=a_{1} e^{i \gamma_{1}}+a_{2} e^{i \gamma_{2}}, \\
& \Phi=\omega_{x} t+\Phi(0), \\
& \omega_{x}=\omega_{x}(0)=\text { const }
\end{aligned}
$$

where $a_{1,2}-$ real oscillation amplitude, $\gamma_{1,2}=\omega_{1,2} t+\gamma_{1,2}(0)-$ phase, $\gamma_{1,2}(0), \Phi(0)$, $\omega_{x}(0)$ - initial values of the variables, $\omega_{1,2}=\bar{J}_{x} \omega_{x} / 2 \pm \omega_{a}-$ oscillation frequency, $\omega_{a}=\sqrt{\bar{J}_{x}^{2} \omega_{x}^{2} / 4+\omega_{0}^{2}}$.

Variables are considered $a_{1,2}, \gamma_{1,2}$, as new variables, and using the method of variation of arbitrary constants, we obtain

$$
\begin{aligned}
& \frac{d a_{1,2}}{d t}=\left[a_{1,2} \dot{\omega}_{1,2}+a_{2,1} \dot{\omega}_{2,1} \cos \left(\gamma_{2}-\gamma_{1}\right)-\varepsilon \operatorname{Im}\left(F e^{-i \gamma_{1,2}}\right)\right] / 2 \omega_{a} \\
& \frac{d \omega_{x}}{d t}=\varepsilon f\left(r, a_{1,2}, \gamma_{1,2}, \omega_{x}, \Phi\right), \quad \frac{d \gamma_{1,2}}{d t}=\omega_{1,2}+\varepsilon \ldots
\end{aligned}
$$

where $\dot{\omega}_{1,2}=\frac{d \omega_{1,2}}{d t}=\frac{\partial \omega_{1,2}}{\partial \omega_{x}} \frac{d \omega_{x}}{d t}+\frac{\partial \omega_{1,2}}{\partial \omega} \frac{d \omega}{d t}$.

Corresponding averaged system (after averaging over phases $\gamma_{1}, \gamma_{2}, \Phi$ ) for the slow variables $a_{1,2}, \omega_{x}$ has the form

$$
\begin{aligned}
& \frac{d a_{1}}{d t}=-\left[a_{1}\left\langle\dot{\omega}_{1}\right\rangle+a_{2}\left\langle\dot{\omega}_{2} \cos \left\langle\gamma_{2}-\gamma_{1}\right\rangle\right\rangle-\varepsilon \operatorname{Im}\left(c_{100}\right)\right] / 2 \omega_{a} \\
& \frac{d a_{2}}{d t}=\left[a_{2}\left\langle\dot{\omega}_{2}\right\rangle+a_{1}\left\langle\dot{\omega}_{1} \cos \left(\gamma_{2}-\gamma_{1}\right)\right\rangle-\varepsilon \operatorname{Im}\left(c_{010}\right)\right] / 2 \omega_{a} \\
& \frac{d \omega_{x}}{d t}=\varepsilon\langle f\rangle
\end{aligned}
$$

where $\langle\cdot\rangle$ - standard averaging operator in phases $\gamma_{1,2}, \Phi ; c_{100}$ and $c_{010}$ - the coefficients of the Fourier series $F\left(r, a_{1,2}, \gamma_{1,2}, \omega_{x}, \Phi\right)$, recorded in complex form; $\operatorname{Im}(\cdot)$ - designation of the imaginary part of the complex quantity.

The system (16)-(18) for simplicity kept the same notation that is not an averaged system.

For symmetrical descent capsule at nominal strength tether tension averaged equations (16)-(17) can be integrated in terms of elementary functions. In this case, we have

$$
\frac{d a_{1,2}}{d t}= \pm \frac{\dot{\omega}_{1,2}}{2 \omega_{a}} a_{1,2}
$$


where $\dot{\omega}_{1,2}= \pm \frac{\omega_{0}}{\omega_{a}} \dot{\omega}_{0}$

Hence,

$$
a_{1,2}=a_{1,2}(0)\left(\frac{\frac{\bar{J}_{x}^{2} \omega_{x}^{2}}{4}+\omega_{0}^{2}(0)}{\frac{\bar{J}_{x}^{2} \omega_{x}^{2}}{4}+\omega_{0}^{2}(r)}\right)^{0.25}
$$

where $\omega_{0}^{2}(0)$ - the initial value of the function $\omega_{0}^{2}(r)$.

If the angular velocity $\omega_{x}$ slowly changes with time, for example due to dissipation in the system, it is included in the vector $r$.

To evaluate the main characteristics of the rotational movement of cargo by the formulas (20), the following relation

$$
\begin{aligned}
& \alpha_{\max }=a_{1}+a_{2}, \quad \alpha_{\min }=\left|a_{1}-a_{2}\right| \\
& \omega_{\max }=\left|a_{1} \omega_{1}+a_{2} \omega_{2}\right|, \quad \omega_{\min }=\left|a_{1} \omega_{1}-a_{2} \omega_{2}\right|
\end{aligned}
$$

where $\alpha_{\max }, \alpha_{\min }$ and $\omega_{\max }, \omega_{\min }-$ evaluation envelope curves for the nutation angle $\alpha=1$ $\xi \mid$ and angular velocity cargo $\omega_{n}=\sqrt{\omega_{y}^{2}+\omega_{z}^{2}}$.

The obtained analytical solution corresponds to the spatial movement of small spacecraft with a given programme change tether tension force $T_{0}(r)$ slowly changing with time.

Figure 3 Time history of nominal tether tension (see online version for colours)

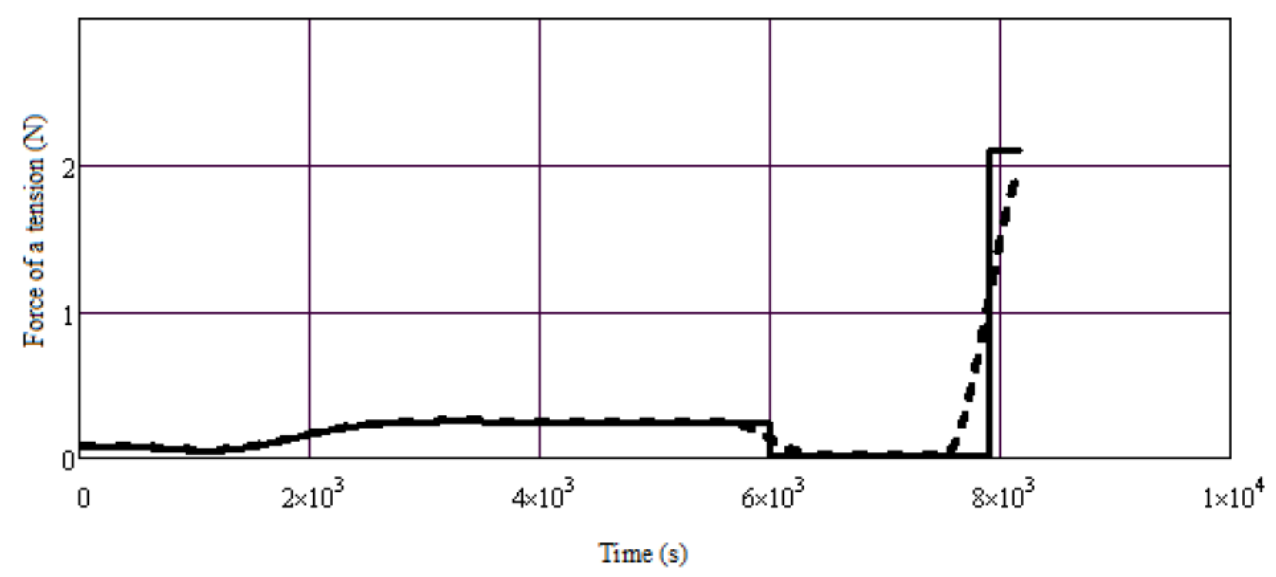

As an example of the use of analytical solutions (21)-(22), we consider the deployment of the programme, which is close to the programme used in the experiment YES2 (Kruijff, 2011). The changing of the nominal tensile force in this case is shown in Figure 3. This figure shows two plots. The solid line corresponds to the relay switching power tension between the individual characteristic areas of deployment. The dashed line shows the dependence, which is smoothed, which ensures the continuity of the first 
derivative of the above function. As shown in the monograph (Zabolotnov, 2013), it is desirable to use the smoothed dependencies for tensile force. Firstly, because the implementation of relay dependencies when the control system is working is difficult due to the inertia of any system; secondly, it allows avoiding abrupt discontinuities of the nutation angle that could cause the angular movement of the cargo to be unstable. Figures 4(a) and 4(b) compare the dependencies $\alpha(t), \omega_{n}(t)$, calculated from the original equations of motion (1)-(4), with estimates for the envelope curves (21)-(22). The comparison was made with the following initial conditions of the rotational movement of the cargo: $\alpha(0)=8^{\circ}, \dot{\alpha}(0)=0, \dot{\psi}(0)=0, \omega_{x}(0)=0.05 \mathrm{~s}^{-1}$. Numerical results show that the analytical solution well describe the angular movement of the cargo at a slow change in the nominal tether tension force and at small angles of nutation (approximately $30-40^{\circ}$ ).

Figure 4 (a) Time history of nutation angle for nominal tether tension

(b) Time history of angular velocity $\omega_{n}=\sqrt{\omega_{y}^{2}+w_{z}^{2}}$ for nominal tether tension (see online version for colours)

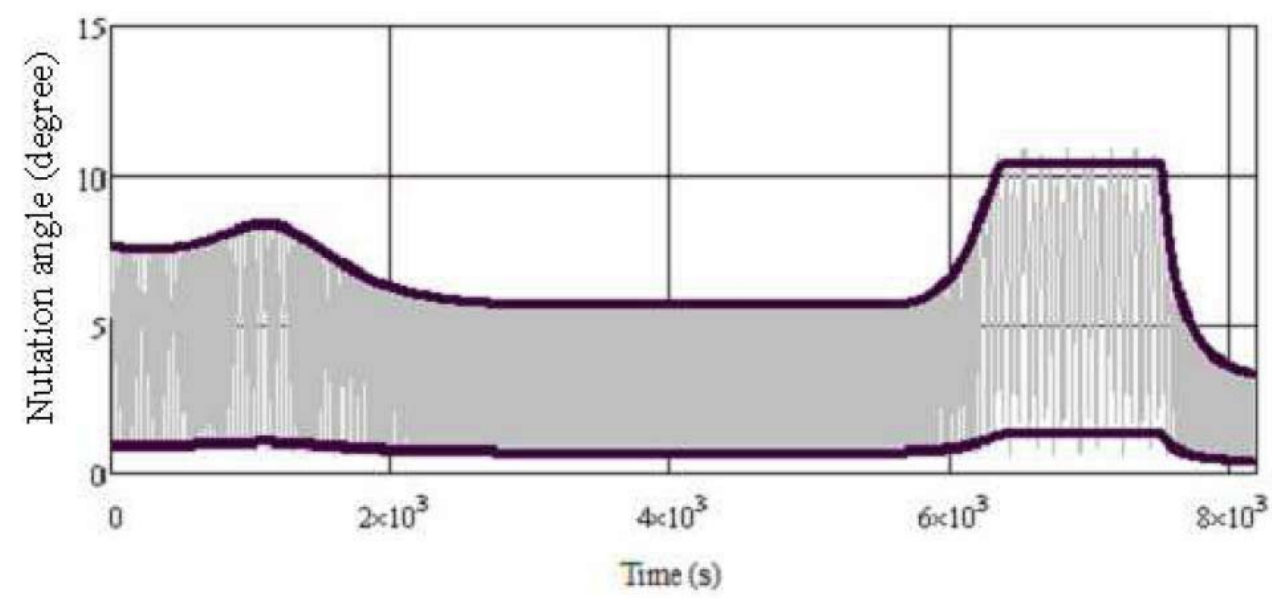

(a)

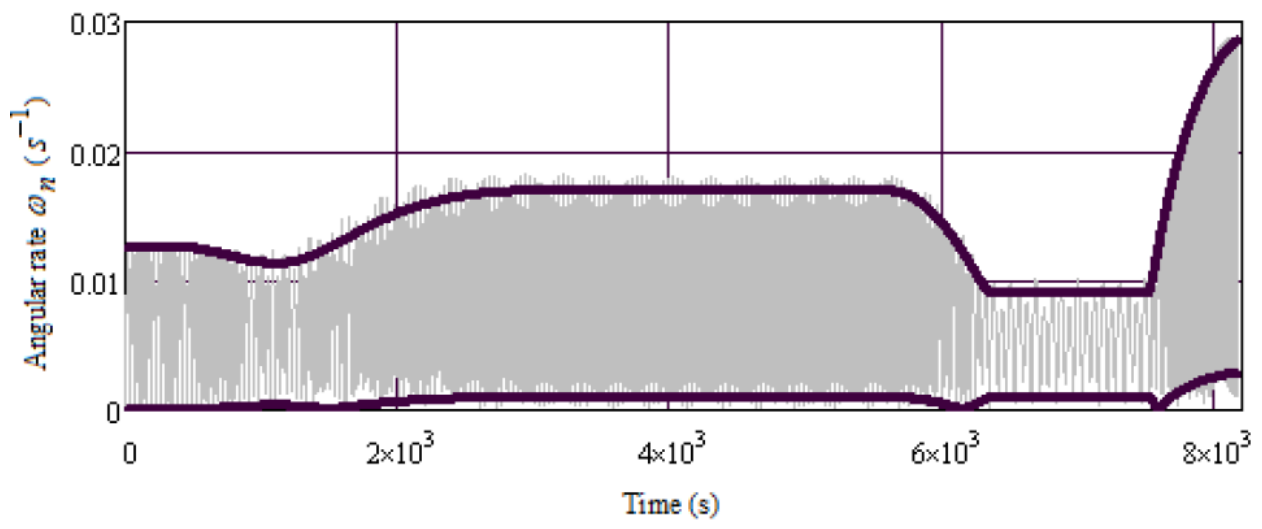

(b) 
Averaging equations (14)-(15) held the non-resonant case. Resonance case requires separate consideration. It should be noted that the averaged equation resonance case is not depend on static and dynamic asymmetries. This indicates that the effect on asymmetry cargo non-resonant portions slightly. The asymmetry is characterised by the dimensionless parameters $\Delta \bar{y}=\Delta y / \Delta x, \Delta \bar{z}=\Delta z / \Delta x, \Delta \bar{J}=\left(J_{z}-J_{y}\right) / J, \bar{J}_{x y}=J_{x y} / J$, $\bar{J}_{x z}=J_{x z} / J, \bar{J}_{y z}=J_{y z} / J$, where $J=\left(J_{z}+J_{y}\right) / 2$. Even with relatively high values of these parameters (about 10\%), the effect of asymmetry in the non-resonant sections can be seen in small 'beats' in the amplitude of oscillation of the nutation angle, which is shown by numerical simulation on the original equations of motion (Zabolotnov and Naumov, 2012; Zabolotnov, 2013).

The influence of asymmetry increases when it hit trajectory in the resonant region. Resonance regions are characterised by the conditions $\omega_{x} \approx \omega_{1,2}$. From these conditions, we can easily obtain the resonance values of the angular velocity of rotation of the cargo on its own axis. Thus, the resonant angular velocity (resonance curves) are functions that vary slowly over time in accordance with a change in the nominal tension force of the tether $T_{n}(r)$, which determines the frequency $\omega_{0}(r)$. Therefore, the conditions for passage of the trajectory in the resonance region can be written in another: $\omega_{x}^{r}(r) \approx \omega_{x}$. After passage of the trajectory of the system in the resonance region nutation angle usually begins to increase rapidly and the use of equations written for small angles of nutation becomes not valid.

In order to apply the asymptotic analysis of the motion of the small spacecraft on a tether at high angles of nutation need a different approach. This approach is based on the method of integral manifolds.

\section{Analysis of the rotational motion of small spacecraft on a tether method of integral manifolds}

At constant tether tension force and the symmetric small spacecraft (if you do not take into account other disturbances) of the projection of the angular momentum $K_{x}, K_{x t}$ is constant, that is, are the integrals of motion. Therefore, to apply the method of integral manifolds of vector dynamic equation (1) can be written in the variables $K_{x}, K_{x t}, \omega_{z n}$, where the angular velocity $\omega_{z n}$ determines the oscillatory movement of the cargo in the plane of the nutation angle (plane $c x y_{n}$, Figure 2). In these variables, dynamic and kinematic Euler equations take the form (Zabolotnov, 2013)

$$
\begin{aligned}
& \frac{\tilde{d} K_{x}}{d t}=\Delta M_{x}^{s}+\Delta M_{x}^{d} \\
& \frac{\tilde{d} K_{x t}}{d t}=\Delta \tilde{M}_{x t} \\
& \frac{d \omega_{z n}}{d t}=\frac{M_{z n}^{t}}{J}+\frac{\left(K_{x} \cos \alpha-K_{x t}\right)\left(K_{x}-K_{x t} \cos \alpha\right)}{J^{2} \sin ^{3} \alpha}+\frac{\Delta M_{z n}^{d}+\Delta M_{z n}^{s}}{J} \\
& \frac{d \alpha}{d t}=\omega_{z n}+\Delta \dot{\alpha}
\end{aligned}
$$




$$
\begin{aligned}
& \frac{d \varphi}{d t}=\frac{K_{x}}{J_{x}}+\frac{K_{x} \cos \alpha-K_{x t}}{J \sin ^{2} \alpha} \cos \alpha+\Delta \dot{\varphi}_{d}+\Delta \dot{\varphi} \\
& \frac{d \psi}{d t}=\frac{K_{x t}-K_{x} \cos \alpha}{J \sin ^{2} \alpha}+\Delta \dot{\psi}_{d}+\Delta \dot{\psi}
\end{aligned}
$$

where $\Delta \dot{\varphi}_{d}, \Delta \dot{\psi}_{d}$ - amendments to derivatives of the Euler angles arising from the presence of a rigid body dynamic asymmetry; $M_{z n}^{t}=-\Delta x T \sin \alpha(\Delta x>0)$ - righting moment on the strength of the tether tension; $\Delta M_{x}^{s}, \Delta M_{z n}^{s}$ - components of the principal vector of torque, depending on the static asymmetry; $\Delta M_{x}^{d}, \Delta M_{z n}^{d}-$ components of the principal vector of torque, depending on the dynamic asymmetry; $\Delta \tilde{M}_{x t}-$ term dependent on the angular velocity of rotation of the tether system of coordinates.

A feature of the system (23)-(28) is that the angle of the precession $\psi$ does not depend on the right-hand side of equations (23)-(27). Therefore, for further analysis, equation (28) cannot be seen, and the change in the precession angle to find after the definition of the corresponding asymptotic solutions for other variables in the system.

For unperturbed decisions are solutions of system (23)-(28), which can be obtained in the absence of asymmetry and tether rotation of the coordinate system for constant tether tension force $T=$ const. Based on this system (23)-(28) introduced a small parameter $\varepsilon$ by scaling the corresponding terms, then

$$
\begin{aligned}
& \frac{d^{2} \alpha}{d t^{2}}+F(\alpha, x)=\varepsilon f(\alpha, \varphi, x) \\
& \frac{d \varphi}{d t}=\omega_{\varphi}(\alpha, x)+\varepsilon \Phi(\alpha, \varphi, x) \\
& \frac{d x}{d t}=\varepsilon X(\alpha, \varphi, x)
\end{aligned}
$$

where $x$ - vector of slow variables of the system,

$$
\begin{aligned}
& F(\alpha, x)=-\frac{M_{z n}^{t}}{J}+\frac{\left(K_{x} \cos \alpha-K_{x t}\right)\left(K_{x t} \cos \alpha-K_{x}\right)}{J^{2} \sin ^{3} \alpha} \\
& \omega_{\varphi}(\alpha, x)=\frac{K_{x}}{J_{x}}+\frac{K_{x} \cos \alpha-K_{x t}}{J \sin ^{2} \alpha} \cos \alpha .
\end{aligned}
$$

In the vector of slow variables are variables that characterise the movement around the centre of mass of the cargo $\left(K_{x}, K_{x t}\right)$, and components, a change which does not depend on the orientation and the angular velocity. The latter values are, for example, the programme changes the tether length and the angle of its deviation from the vertical, and other such variables that determine the angular velocity of rotation of the tether system of coordinates. Therefore, $x=\left(K_{x}, K_{x t}, r\right)$, where $r$ - vector of slow variables characterising the programme deployment STS. 
The introduction of a small parameter in the system of equations (29)-(31) is in the nature of the hypothesis, the validity of which is already checked when dealing with specific examples where known deployment programme, the range of asymmetries, etc. Deployment programme STS is characterised by dependence $T(r)$. In this case, the dependence of the tether tension on the vector $r$ is similar to the dependence on the slow time. Construction of asymptotic solutions of the system (29)-(31) is made taking into account the principal resonance $\omega_{\varphi}(\alpha, x)=0$ (resonance of the lowest order). In this case, the approximate equations are described as non-resonant motion of the cargo, and in the vicinity of the principal resonance. To do this, the system (29)-(31) entered the slow time $\tau=\mu t$, where $\mu=\varepsilon^{0.5}$, and the normalised distance to the resonant surface $\rho(\alpha, x)=$ $\mu^{-1} \omega_{\varphi}(\alpha, x)$. In this case, the system (29)-(31) is to the form

$$
\begin{aligned}
& \mu \frac{d \omega \alpha}{d \tau}+F(\alpha, x)=\mu^{2} f(\alpha, \varphi, x) \\
& \mu \frac{d \alpha}{d \tau}=\omega \alpha \\
& \frac{d \varphi}{d \tau}=\rho(\alpha, x)+\mu \Phi(\alpha, \varphi, x) \\
& \frac{d x}{d \tau}=\mu X(\alpha, \varphi, x)
\end{aligned}
$$

The technique for constructing asymptotic solutions for systems of the form (32)-(35), the method of integral manifolds is given in Zabolotnov and Lyubimov (2003). $(\mu=0)$

Singularly perturbed equations (33)-(36) corresponds to the degenerate system

$$
F\left(\alpha_{0}, x_{0}\right)=0, \quad \omega_{\alpha 0}=0, \quad \frac{d \varphi_{0}}{d \tau}=\rho\left(\alpha_{0}, x_{0}\right), \quad \frac{d x_{0}}{d \tau}=0
$$

It is assumed that the equation $F\left(\alpha_{0}, x_{0}\right)=0$ is isolated root $\alpha_{0}\left(x_{0}\right)$, and for this root

$$
\frac{\partial F}{\partial \alpha}>0
$$

Within the variables $\alpha_{0}, \omega_{\alpha_{0}}, \varphi_{0}, x_{0}$, the relations (36) define the integral manifold whose vicinity is used to construct asymptotic solutions.

Adjoint system method of integral manifolds in this case has the form

$$
\frac{d^{2} \tilde{\alpha}}{d t^{2}}+F\left(\tilde{\alpha}, x_{0}\right)=0
$$

and determines the fluctuations of the nutation angle in the vicinity of the integral manifold.

If we determine the derivative $\frac{\partial F}{\partial \alpha}$ and find the nutation angle of from the equation $F\left(\alpha_{0}, x\right)=0$, it is easy to get 


$$
\frac{\partial F}{\partial \alpha}\left(\alpha_{0}\right)=\frac{\left(K_{x}^{2}+K_{x t}^{2}-2 K_{x} K_{x t} \delta\left(\alpha_{0}\right)\right)}{J^{2} \sin ^{4} \alpha_{0}}\left(3 \cos ^{2} \alpha_{0}+1\right)
$$

where $\delta\left(\alpha_{0}\right)=\frac{\cos ^{2} \alpha_{0}+3}{3 \cos ^{2} \alpha_{0}+1} \cos \alpha_{0}$ and $\left|\delta\left(\alpha_{0}\right)\right|<1$. Therefore, the condition (37) holds for $\alpha_{0} \in(0, \pi)$.

Asymptotic solutions of the system (32)-(35) in the vicinity of the integral manifold sought in the form

$$
y=\bar{y}+y^{(n)}, \quad z=\bar{z}+z^{(n)}
$$

where $y=(x, \varphi), z=\left(\alpha, \omega_{\alpha}\right), \bar{y}=y_{0}(\tau)+\mu y_{1}(\tau)+\mu^{2} \ldots$ and $\bar{z}=z_{0}(\tau)+\mu z_{1}(\tau)+\mu^{2} \ldots-$ series are defining the slow motion of the system; $y^{(n)}=y_{0}^{(n)}(t)+\mu y_{1}^{(n)}(t)+\mu^{2} \ldots$ and $z^{(n)}=z_{0}^{(n)}(t)+\mu z_{1}^{(n)}(t)+\mu^{2} \ldots-$ series defining the slow motion of the system.

Then, in accordance with the method used (Zabolotnov and Lyubimov, 2003), slow motion in the vicinity of the integral manifold is determined by the system

$$
\begin{aligned}
& \frac{d \bar{\varphi}}{d \tau}=\rho(\bar{\alpha}, \bar{x})+\mu \Phi(\bar{\alpha}, \bar{\varphi}, \bar{x}) \\
& \frac{d \bar{x}}{d \tau}=\mu X(\bar{\alpha}, \bar{\varphi}, \bar{x})
\end{aligned}
$$

where $\bar{\alpha}=\alpha_{0}(\tau)+\mu \alpha_{1}(\tau)+\mu^{2} \ldots$

From the point of view of the problem of the slow component of the solution $\bar{y}, \bar{z}$ is characterised by the precessional motion of the cargo, and the fast component of the solution $y^{(n)}, z^{(\mathrm{n})}$ describe its fluctuations in the plane of the nutation angle. Quick solutions correspond to boundary components of the members in the theory of singularly perturbed systems.

If we consider the case of small amplitude of nutation oscillations cargo, the behaviour of the members of the border for an angle $\alpha$ are described by the equation of the second order

$$
\frac{d^{2} \alpha^{(n)}}{d t^{2}}+\omega_{b}^{2} \alpha^{(n)}=F_{2}
$$

Here $\omega_{b}^{2}=\sqrt{\partial F / \partial \alpha}$, where the derivative $\partial F / \partial \alpha$ is defined by $\alpha=\alpha_{0}\left(x_{0}\right), F_{2}-$ perturbing function.

For statically and dynamically symmetric cargo the original equations become simpler due to the fact that the angle of proper rotation is independent of other variables. Then the asymptotic solution of (29)-(31) are constructed directly in the small parameter $\varepsilon$, singularly perturbed system takes the form

$$
\varepsilon^{2} \frac{d^{2} \alpha}{d \tau^{2}}+F(\alpha, x)=\varepsilon f(\alpha, x)
$$




$$
\frac{d x}{d \tau}=X(\alpha, x)
$$

Asymptotic separation of motions in the system (44)-(45) is made similarly, by the method described above, for a more general system. As an example, consider the simplest case when the programme deployment STS is given as a function of the slow variables $T(r)$.

The movement on the integral manifold in this case is described by a nonlinear equation

$$
F(\alpha, x)=\frac{M_{z n}^{t}}{J}+\frac{\left(K_{x} \cos \alpha-K_{x t}\right)\left(K_{x t} \cos \alpha-K_{x}\right)}{J^{2} \sin ^{3} \alpha}=0
$$

Not to solve the nonlinear equation (46) at each time point, we can differentiate the implicit function (46) and obtain a differential equation for the slow component of the solution for the nutation angle

$$
\frac{\partial F}{\partial \alpha} \frac{d \alpha}{d t}=-\frac{\Delta x \sin \alpha}{J} \frac{d T}{d t}
$$

where the derivative $d T / d t$ is determined by virtue of the selected programme deployment STS. Then, it is necessary to solve equation (46) only at the initial time.

Quick small vibrations in the plane of the nutation angle are described by the equation of the second order (43), where $F_{2}=0$ for this case. Then equation (43) has the classic look of an oscillator with a slowly varying frequency. In order to solve it, Wentzel-Kramers-Brillouin (WKB) method can be used. Zero approximation WKB method for the equation (43) has the form

$$
\alpha^{(n)}(t)=A_{n} \sin \vartheta(t)
$$

where $A_{n}$ is the amplitude of the fast nutation oscillations depending on the slowly changing variables; $\vartheta(t)$ is phase of the oscillations.

The amplitude and phase of rapid nutation oscillations are determined by the approximate formulas

$$
A_{n}=\sqrt{\frac{C}{\omega_{b}}}, \quad \vartheta(t)=C_{1}+C \int_{0}^{t} \frac{d t}{A_{n}^{2}(t)}
$$

where arbitrary constants $C$ and $C_{1}$ are determined from the initial conditions.

$$
C=\omega_{b}\left[\alpha^{(n)}(0)\right]^{2}+\frac{1}{\omega_{b}}\left[\frac{d \alpha^{(n)}}{d t}(0)\right]^{2}, \quad C_{1}=\vartheta(0)=\operatorname{arctg}\left(\frac{\omega_{b} \alpha^{(n)}(0)}{\frac{d \alpha^{(n)}}{d t}(0)}\right)
$$


Figure 5 (a) Time history of nutation angle (the case of direct precession) (b) Time history of precession angle derivative $d \psi / d t$ (the case of direct precession)

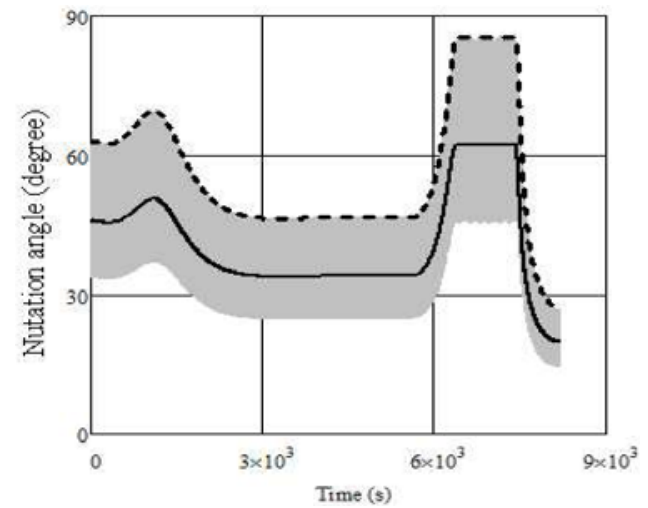

(a)

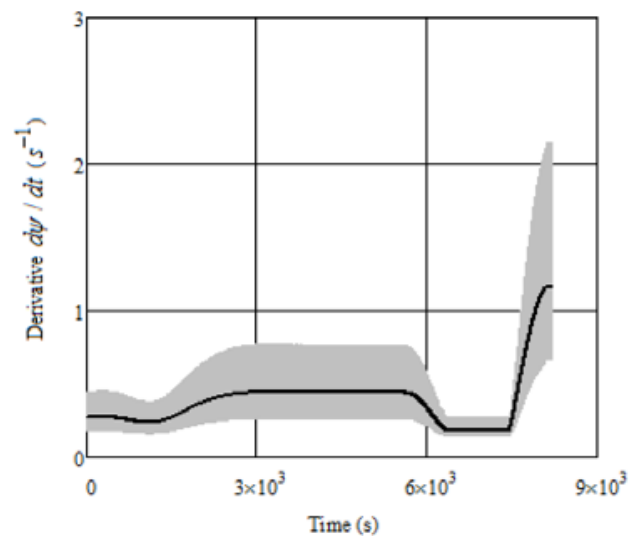

(b)

Depending on the initial conditions at the time of detaching of the cargo, which are random variables, various cases of its spatial movement can be implemented. This may be a 'direct' precession, when the signs of $d \psi / d t$ and $\omega_{x}$ are the same, and 'reverse' precession, when the signs of $d \psi / d t$ and $\omega_{x}$ are opposite. Figure 5, as an example, shows the dependence of the nutation angle and derivative $d \psi / d t$ of time for the case of 'direct' precession with the initial conditions $\omega_{x}(0)=0.02 \mathrm{~s}^{-1}, K_{x t} / J=0.15 \mathrm{~s}^{-1}$. The initial nutation angle for precession equation (47) is then determined from the solution of the nonlinear equation (46), the initial nutation angle for the original equations was different from the precession angle by $0.3 \mathrm{rad}$. Solutions corresponding to the initial equations are a rapid oscillation of relatively slow components of the solution (thick lines). Figure 5 also shows the envelope for the nutation angle (dotted line), calculated using the analytical solution (48). In this example, the cargo is a sphere of radius $0.2 \mathrm{~m}$ and mass $20 \mathrm{~kg}$ with several prolate ellipsoid of inertia $J_{x} / J=0.8$. These approximate solutions have shown a fairly good agreement between the results from the original model to evaluate the envelope of the nutation angle in a wide range of parameters $J_{x} / J$ and nutation angle (nutation angle for the slow component of the solution can reach $\pi / 2$ ). 
There was only a slight increase in the error in the evaluation of the oscillation amplitudes of the nutation angle to the body with a highly prolate ellipsoid of inertia. For example, when $J_{x} / J=0.1$, the error is $1-3^{\circ}$. It should be also noted that if the deployment programme for STS tether tension force is given as an explicit function of time, the method under consideration for small fast nutation oscillations reduces the problem of estimating the values of the nutation angle to the use of only nonlinear equation (46) and the analytical solution (48)-(49).

These results show that when the cargo on the tether symmetrical about the centre of mass, there are at least two characteristic time scale. The first time scale corresponds to the slow motion of the system and is determined by the time-dependent tether tension force, the second time scale corresponds to the rapid nutation oscillations with respect to the slow component of the solution. Considered time scales are characterised by the corresponding rates of change of variables in the system. The velocity of change in the slow variables is characterised by the parameter $v_{s}=\left|\frac{1}{T} \frac{d T}{d t}\right|$. For quick movement characteristic parameter can take the frequency $v_{f}=\omega_{b}$. The ratio of these variables is a quantity proportional to the small parameter of the problem. Of course, this value is variable in the process of deploying a tether system, but still it can serve as a measure of the assumptions that have been taken at the beginning and which yielded in terms of numerical modelling is an acceptable result for the asymptotic separation of motions of a rigid body. For the example shown in Figure 5, the parameter $v_{f} / v_{s}$ is less than 0.1, and the maximum for this parameter are observed in the transition from the first stage to the second deployment (weakening of the tension force) and under heavy braking the tether on the second portion of the deployment.

\section{Analysis of the resonant modes of motion of the small spacecraft on a tether}

Resonance phenomena in the motion of the asymmetric cargo on the tether arise at the intersection of the trajectory of the resonance regions, which in this case is determined from the condition $\omega_{x}^{r}(r) \approx \omega_{x}$, where the resonance angular velocity is determined for the linear case. The method of integral manifolds is allowed to specify the values of the resonance angular velocities for considerable angles of nutation. For this, it is necessary to express the angular velocity $\omega_{x}=K_{x} / J_{x}$ of the conditions $\omega_{\varphi}(\alpha, x) \approx 0$, the variable $K_{x t}$ determine on the integral manifold (46). Then

$$
\omega_{x}^{r}(r, \alpha)= \pm \sqrt{\frac{\Delta x T(r) \cos \alpha}{J-J_{x}}}
$$

where it is used for the slow component of the nutation angle.

Thus, if the cargo has a prolate ellipsoid of inertia $J_{x}<J$, the resonant modes of motion can exist at $0<\alpha<\pi / 2$, for the opposite case, when $J_{x}>J-$ when $\pi / 2<\alpha<\pi$. If the body is close to the field $J_{x} \approx J$ and especially not twisted, then the condition $\omega_{x} \approx \omega_{x}^{r}(r, \alpha)$ can be satisfied, or if the nutation angles close to $\pi / 2$, or at small values of tether tension force $T(r)$. In general, the expression (50) defines a space of variable $(x, \alpha)$ two symmetric resonance curves crossing which may cause resonance effects. It is also 
necessary to note the presence of asymmetry in the angular velocity $\omega_{x}$ is not constant, and there is a theoretical possibility of attraction to the resonance curve, even for cargo ellipsoid of inertia of the form $J_{x}>J$ (Zabolotnov and Lyubimov, 1998).

Figure 6 (a) Time history of angular rate $\omega_{x}$ and $\omega_{x}^{r}$ (resonant angular rate) (b) Time history of nutation angle (disturbance at pass through a resonance)

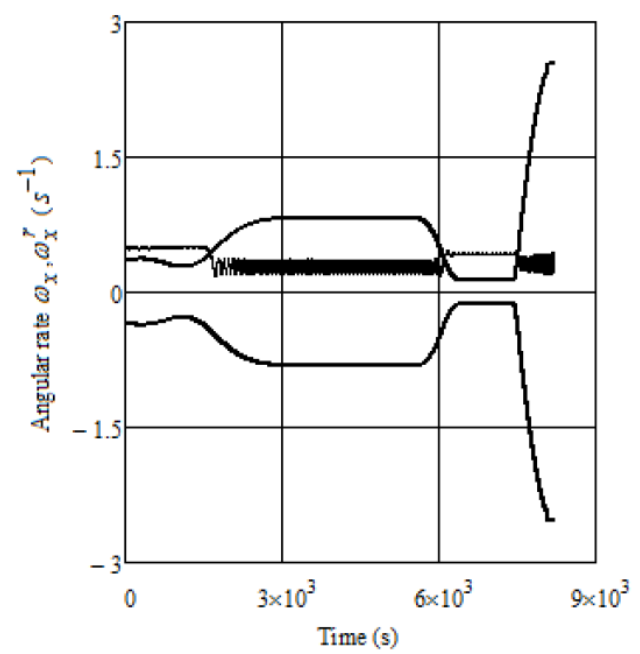

(a)

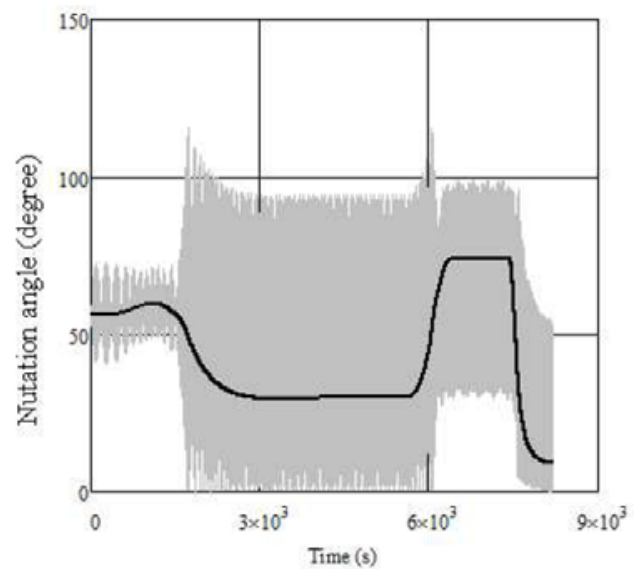

(b)

When crossing the resonance curves there are two possible scenarios of behaviour of trajectories of the system: the passage through resonance and implementation of a long enough near-resonance mode of motion, when for some finite period of time $d \varphi / d t \approx 0$. It is clear that in the latter case, the effect of the asymmetry of a rigid body in its motion increases. Figure 6 shows plots illustrating the effect of the asymmetry of a rather simple form (static asymmetry) $\Delta y / \Delta x=0.1$ on the movement of cargo, which are symmetrical about the x-axis curves, correspond to the resonant angular velocities (50) and are defined on the integral manifold. In this example, there are implemented three passes through 
resonance, which leads to sharp changes in the angular velocity $\omega_{x}$ and the amplitudes of oscillation of the nutation angle $\alpha$. The bold line in Figure 6(b) shows a slow component of the solution for the angle $\alpha$, at which the resonance curves were determined. In this example, the programme is used to deploy STS, shown in Figure 3, and the following parameter $J_{x} / J=0.8, \omega_{x}(0)=0.5 \mathrm{~s}^{-1}, K_{x t} / J=0.15 \mathrm{~s}^{-1}$.

Despite the fact that the nutation angle varies within a wide range (passes through resonance lead to violation of limit $\alpha<90^{\circ}$ ), the time of passing through resonance is correctly predicted by resonance curves determined in the integral manifold, although dependencies $\omega_{x}(t)$ and $\alpha(t)$ are calculated by the original model of the motion of a small spacecraft [(23) to (28)]. The possible implementation of long resonant modes of cargo movement on the tether at no small angles of nutation and the presence of mass-inertial asymmetry is probabilistic in nature and requires a separate study.

\section{Effect of the release tether management system on the stability of the angular movement of the cargo}

Tether tension force in the implementation of programmes to deploy STS differs from nominal power due to operation of the control system of a tether release. Functioning of the control system is characterised by a transient, the quality of which depends on many factors (choice of feedback coefficients, inertia control mechanism, etc.). Furthermore, when constructing the nominal deployment programmes generally do not include tether elongation and other characteristics. Therefore, the tensile force of the tether can be formally represented as a sum (6) $T=T_{n}+\Delta T$. The resulting perturbation acting on the angular movement of cargo is like a parametric action since it leads to a change in the oscillation frequency in the plane of the nutation angle. It can be seen, for example, if in equation (10) instead of a specific oscillation frequency $\omega_{0}(r)$, for the unperturbed motion treat rate to account for this disturbance: $\omega=\sqrt{\Delta x\left(T_{n}+\Delta T\right) / J}$. It is understood that the frequency spectrum of the perturbation depends on the above mentioned factors (coefficients of feedback characteristics of a tether, etc.). It is known that a periodic perturbation parameter acting on an oscillating system, can lead to loss of its stability properties (Mathieu equation). These considerations are confirmed by numerical examples of modelling the angular motion of a small spacecraft at deployment of STS, which are presented below.

In this case, the equations of motion STS conveniently written in the geocentric Cartesian coordinate system associated with the plane of the orbital motion of the centre of mass STS. In the simplest case, a model of two material points connected by a weightless tensile bond. This model, despite its simplicity, has been used for many tasks (Beletski and Levin, 1990), then the equations of motion can be written in the form of STS

$$
\frac{d \vec{r}_{k}}{d t}=\vec{V}_{k}, \quad m_{k} \frac{d \vec{V}_{k}}{d t}=\vec{G}_{k}+\vec{T}_{k}
$$

where $k=1,2$ - indices corresponding to the cargo and the basic spacecraft; $\vec{r}_{k}, \vec{V}_{k}-$ the radius vector and velocity in the geocentric coordinate system; $m_{k}-$ mass of 
bodies; $\quad \vec{G}_{k}=-K \frac{m_{k} \vec{r}_{k}}{r_{k}^{3}}-$ gravitational forces in the central field of the Earth; $\vec{T}_{2}=T \frac{\vec{r}_{1}-\vec{r}_{2}}{\left|\vec{r}_{1}-\vec{r}_{2}\right|}\left(\vec{T}_{1}=-\vec{T}_{2}\right)-$ force acting on the cargo and the spacecraft respectively; $T$ - module of elasticity force.

Since the tether is not in compression, the elastic force unit is computed according to the law of Hooke expression

$$
T= \begin{cases}c \frac{\left|\vec{r}_{1}-\vec{r}_{2}\right|-L}{L}, & \text { if }\left|\vec{r}_{1}-\vec{r}_{2}\right|-L \geq 0 \\ 0, & \text { if }\left|\vec{r}_{1}-\vec{r}_{2}\right|-L<0\end{cases}
$$

where $L$ - unstretched length released from the mechanism of the tether, $c=E A-$ modulus of elasticity, $E$ - Young's modulus, $A=\pi D^{2} / 4-$ cross sectional area of the tether, $D$ - tether diameter.

These equations must be an attached equation operation of the brake mechanism. In the simplest case, when a change in inertia is neglected management ( $m_{i}=$ const), these equations have the form (Zabolotnov and Naumov, 2012)

$$
m_{i} \frac{d V_{L}}{d t}=T-F_{c}, \quad \frac{d L}{d t}=V_{L}
$$

where $L$ and $V_{L}$ - unstretched length of tether and its velocity of release, $F_{c}-$ control force, $m_{i}$ - mass characterising the inertia control mechanism. In accordance with the principle of force $F_{c}$ feedback given in the form

$$
F_{c}=F_{c n}+p_{L} \Delta_{L}+p_{V} \Delta V_{L}
$$

where $p_{L}, p_{V}$ - feedback coefficients, $F_{c n}-$ nominal driving force, $\Delta L=L-L_{n}$, $\Delta V=V-V_{n}-$ control error. If inertia is neglected $m_{i} \rightarrow 0$, it is believed $T=F_{c n}$ and elongation tether neglected.

Modelling of controlled motion STS using the above equations corresponds to the so-called ideal controller, since it does not take into account many of the features of the control system (discrete, delay, etc.). However, a rough estimate of such models is quite suitable. If the system is unstable with perfect control, then the simulation of controlled motion in more complex models of sustainability cannot speak.

In this model, we can estimate the influence of parameters of the control system, the inertia of the brake and the extensibility of the tether on the deployment of tether system. Without considering the problem as a whole, we gave examples of the instability of the angular motion of the small spacecraft with an unhappy choice of the feedback coefficients $p_{L}, p_{V}$. Particularly sensitive to this point of view the initial portion of the deployment (immediately after the removal of the goods), and (for software deployment, corresponding to Figure 3) plot intensive acceleration and deceleration of cargo (the second stage of the deployment in the mission YES2). 
Figure 7 shows an example of the loss ustochivosti angular movement of cargo during unfortunate choice of the feedback coefficients (they are too large: $p_{L}=1.5$, $\left.p_{V}=1.4\right)$. Figure 7(b) shows the dependence tension force from time to time, which shows the areas of tether slack. At these sites the rotation cargo is inertial and after a few such sites buckling of its angular motion, and nutation angle reaches about 180 degrees Figure 7(a). However, if the value of feedback coefficients is small enough $\left(p_{L}=0.005\right.$, $p_{V}=0.4$ ) that the angular movement of the capsule with the same input data is stable and the angle of nutation of the initial section is not more than 10 degrees (no tether slack portions). The graphs shown in Figure 7, constructed with the following initial data: $E S=7,069 \mathrm{~N}, m_{u}=0.6 \mathrm{Kg}, \alpha(0)=8^{\circ}, \omega_{n}(0)=0, \omega_{x}(0)=0.05 \mathrm{~s}^{-1}$.

A similar effect is characterised by the instability of the motion for the second stage deployment STS (Figure 3, $t>6,000 \mathrm{~s}$ ). Such example in article (Ishkov and Zabolotnova, 2010) is considered.

Figure 7 (a) Time history of nutation angle (instability in the initial portion of a tether deployment) (b) Time history of tension force with patches tether slack (see online version for colours)

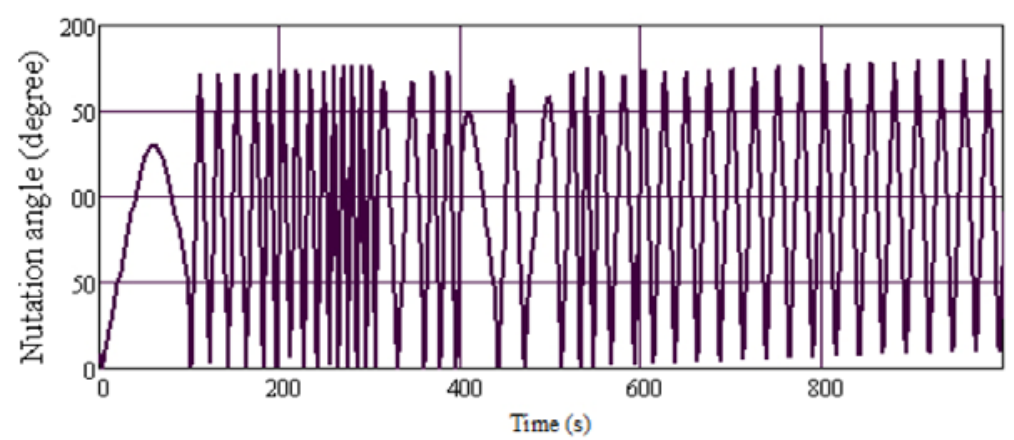

(a)

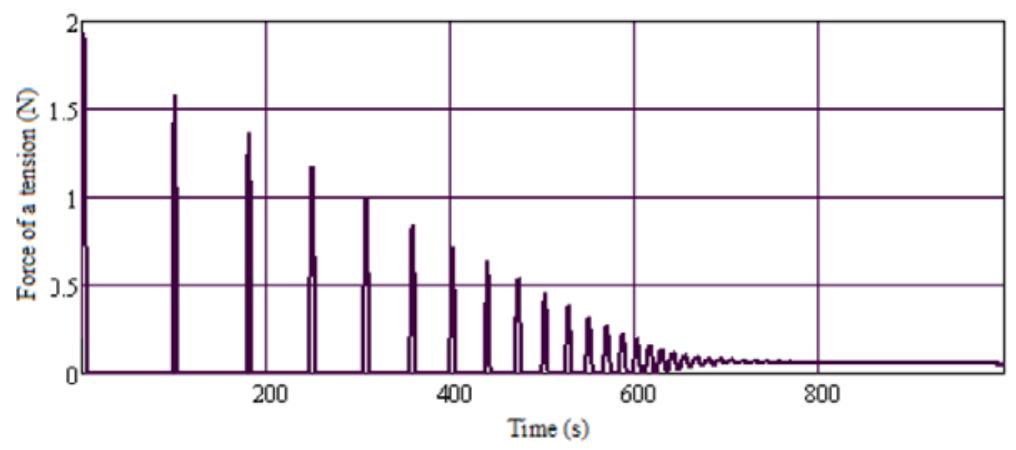

(b) 


\section{Conclusions}

In this article, we systematised and analysed the main factors leading to instability of motion of a small spacecraft with respect to the centre of mass at STS deployment. These factors include:

1 abrupt changes in the nominal tether tension force

2 static and dynamic asymmetry cargo recurrent rise to resonant modes of motion

3 the initial error cargo compartment

4 a bad choice of the feedback coefficients in the regulation of the release of the tether in the initial part deployment system (after the removal of the goods)

5 regulation unintended release tether on the stage of rapid deployment STS, consisting of portions of acceleration and deceleration.

All of these factors must be considered when designing space missions, for transporting cargo via STS. For this, there can be used mathematical models of STS movement and analyses presented in the article.

\section{References}

Beletski, V.V. and Levin, E.M. (1990) Dynamics of Space Tether Systems, in Russian, Science Press, Moscow.

Bogolyubov, N.N. and Mitropolsky, Y.A. (1963) Asymptotic Methods in the Theory of Nonlinear Oscillations, Science Press (in Russian), Moscow.

Hirsh, M., Pugh, C. and Shub, M. (1977) Invariant Manifolds, Berlin, Springer.

Ishkov, S.A. and Zabolotnova, O.Yu. (2010) 'The decision of a problem of stabilization of program expansion orbital tether systems with the account of restrictions on a rotary motion of a trailer body', Bulletin of Samara State Aerospace University, Vol. 21, No. 1, pp.52-61 (in Russian).

Kruijff, M. (2011) Tethers in Space, Uitgeverij BOX Press, Oisterwijk.

Mitropolsky, Y.A. and Lykova, O.B. (1973) Integral Manifolds in the Nonlinear Mechanics, Science Press (in Russian), Moscow.

Nayfeh, A.H. (1973) Perturbation Methods, Wiley, New York.

Tyc, G. and Han, R.P.S. (2001) 'On the dynamics of spinning tethered space vehicles', Philosophical Transactions: Mathematical, Physical \& Engineering Sciences of the Royal Society, Vol. 359, No. 1788, pp.2161-2190, DOI: 10.1098/rsta.2001.0881.

Vigneron, F.R., Schultz, F., Jablonski, A.M. and Tyc, G. (2000) 'Tether deployment and trajectory modeling for space plasma science missions', Journal of Spacecraft and Rockets, Vol. 37, No. 1, pp.78-85, DOI: 10.2514/2.3529.

Zabolotnov, Y. (2013) Introduction of Space Tether System Motion Dynamics and Control, Science Press, Beijing.

Zabolotnov, Y. and Lyubimov, V.V. (1998) 'Secondary resonance effect in the motion of a spacecraft in the atmosphere', Cosmic Research, Vol. 36, No. 2, pp.481-487.

Zabolotnov, Y. and Lyubimov, V.V. (2003) 'Application of the method integral of manifolds for construction of resonant curves for the problem of spacecraft entry into the atmosphere', Cosmic Research, Vol. 41, No. 5, pp.481-487.

Zabolotnov, Y.M. (1994) 'Asymptotic analysis of quasi-linear equations of the motion of a slightly asymmetric spacecraft in the atmosphere I', Cosmic Research, Vol. 31, No. 6, pp.620-629. 
Zabolotnov, Y.M. and Naumov, O.N. (2012) 'Motion of a descent capsule relative to its center of mass when deploying the orbital tether system', Cosmic Research, Vol. 50, No. 2, pp.177-187, DOI: 10.1134/S0010952512020098.

Zhong, R. and Zhu, Z.H. (2013) 'Optimal trajectory design of a deorbiting electrodynamic tether system', Int. J. Space Science and Engineering, Vol. 1, No. 2, pp.128-141. 